\title{
Aproximación a la investigación en el Grado en Medicina de la Universidad de Oviedo
}

\author{
Begoña Cantabrana, Javier Bordallo-Landa, Manuel Sánchez-Fernández, Agustín Hidalgo
}

Objetivo. Presentar iniciativas de fomento de la investigación científica realizadas en el primer curso del Grado en Medicina. Sujetos y métodos. Participaron 150 estudiantes de primer curso que recibieron lecciones teóricas (12 horas, en grupos de 75) y cuatro sesiones (6 horas y 45 minutos) de seminarios sobre descubrimientos científicos, análisis de publicaciones y diseño experimental, en grupos de 4-5. Las tareas de los seminarios estuvieron disponibles con 7-15 días de antelación y, sobre ellas, debían elaborar una presentación para las sesiones y un documento escrito. Ambas actividades fueron objeto de calificación. La evaluación de la satisfacción de los estudiantes se realizó mediante una encuesta.

Resultados. Las tareas relacionadas con descubrimientos científicos se refirieron a aspectos relevantes de la fisiología, la patología, la terapéutica y los desarrollos tecnológicos aplicados a la medicina. También, han analizado y discutido publicaciones científicas y han elaborado propuestas de diseños de investigación a partir de textos breves facilitados por el profesor. Las calificaciones globales obtenidas, sobre cuatro puntos, han sido: 3,71 $\pm 0,07$ y 3,9 $\pm 0,3$ en las tareas sobre descubrimientos y comentarios de artículos científicos, respectivamente, y 3,6 $\pm 0,2$ en el diseño experimental. El $84 \%$ de los matriculados cumplimentaron la encuesta que reflejó una opinión favorable (sobre cinco puntos) de los contenidos teóricos $(4,01 \pm 0,07)$, seminarios sobre descubrimientos $(3,97 \pm 0,1)$ y diseño experimental $(3,87 \pm 0,1)$.

Conclusiones. Los estudiantes tienen una opinión favorable de las actividades realizadas y consideran que pueden contribuir a la formación de los médicos en investigación.

Palabras clave. Educación médica. Formación en investigación. Grado en Medicina. Habilidades de investigación.

\section{Approach to research in the Degree of Medicine of the University of Oviedo}

Aim. To present initiatives for the promotion of scientific research in the Degree of Medicine.

Subjects and methods. The participants included 150 first-year students who received theoretical lessons ( 12 hours, in groups of 75) and, in groups of 4-5, four sessions ( 6 hours and 45 minutes) of seminars on scientific discoveries, publication analysis and experimental design. The topics of seminars were available 7-15 days in advance on which students had to prepare a presentation, and a written document. Both activities were scored. The evaluation of student satisfaction was carried out through a survey.

Results. The tasks were related to scientific discoveries and referred to relevant aspects of physiology, pathology, therapeutics and technological developments applied to medicine. Students also analyzed and discussed scientific publications and prepared research design proposals based on short texts provided by the lecturer. The marks obtained, out of four points, were $3.71 \pm 0.07$ and $3.9 \pm 0.3$ on the tasks of discoveries and comments of scientific articles, respectively, and $3.6 \pm 0.2$ in the experimental design. $84 \%$ of those enrolled completed the survey that indicated a favorable opinion (out of five points) on the theoretical contents $(4.01 \pm 0.07)$ and seminars on discoveries $(3.97 \pm 0.1)$ and experimental design $(3.87 \pm 0.1)$.

Conclusions. The students had a favorable opinion on the activities and considered that they could contribute to the training of research doctors.

Key words. Degree of Medicine. Medical education. Research skills. Research training.

\section{Introducción}

En plena revolución científico-técnica aplicada a la salud, el médico debe tener una sólida formación científica. Así lo reconoce la Organización Médica Colegial en su definición de profesión, en la que afir- ma que, para el desempeño de sus tareas, el médico se ocupará, entre otras actividades, de la producción, el uso y la transmisión del conocimiento científico [1]. Así mismo, las leyes 14/1986 General de Sanidad [2], 16/2003 de Cohesión y Calidad del Sistema Nacional de Salud [3], 44/2003 de Ordenación
Área de Farmacología (Departamento de Medicina). Universidad de Oviedo. Instituto Universitario de Oncología del Principado de Asturias. Obra Social CajAstur. Instituto de Investigación Sanitaria de Asturias. Oviedo, Asturias, España.

Correspondencia: Prof. Agustín Hidalgo Balsera. Área de Farmacología. Departamento de Medicina. Facultad de Medicina y Ciencias de la Salud. Universidad de Oviedo. Julián Clavería, 6. E-33006 Oviedo (Asturias).

E-mail: hidalgo@uniovi.es

Recibido: 24.07.18.

Aceptado: 27.07.18.

Conflicto de intereses: No declarado.

Competing interests: None declared.

(c) 2018 FEM 
Tabla I. Aspectos relacionados con la investigación comentados en las lecciones teóricas.

1. Concepto de ciencia y de conocimiento. Criterios que debe reunir una actividad para ser considerada científica. Tipos de conocimiento. Características del conocimiento científico

2. Ciencia, pseudociencia y mala ciencia. Características diferenciales

3. Tipos de ciencia. ¿Es la medicina una ciencia? La medicina como ciencia natural y social. Componentes científicos de la medicina. Limitaciones de la medicina como ciencia. Medicina como ciencia posnormal

4. Revoluciones científicas en medicina. Concepto de revolución. Revoluciones en el paso del mito a la molécula. Concepto de enfermedad molecular y medicina molecular. Modelo biomédico de investigación. Limitaciones de la medicina basada en pruebas. Finalización de la ciencia

5. Proceso de investigación. Componentes y contextos de la elaboración científica. Investigación fundamental e investigación clínica. Tipos de investigación clínica

6. Metodología de la investigación 1. El contexto de los descubrimientos. Concepto de método y sus características generales y etapas del mismo. El tema de investigación, provocar observaciones. Creatividad. Concepto y tipo de hipótesis

7. Metodología de la investigación 2. El contexto de verificación. Concepto de investigación, investigador y observador. Razones por las que es necesario hacer experimentos. Clasificación de las investigaciones en el laboratorio y en clínica humana. Limitaciones de la investigación en modelos animales; la extrapolación a la clínica. Conceptos de resultados, discusión y modelos de explicación científica 7.1. Modelos de investigación en medicina. Ejemplos paradigmáticos

7.2. Ética de la investigación en medicina. Ética de la utilización de animales de laboratorio. Fundamentos éticos de la investigación clínica en humanos. Regulación legal: consentimiento informado, comités de ética. Nuevos conflictos éticos generados por el avance de la ciencia. Bioética. Repensar los límites de la medicina

8. Metodología de la investigación 3. El contexto de divulgación científica. Vehículos de comunicación científica. Artículos originales, divulgación y vulgarización propiamente dichas

9. Organización de la investigación y políticas científicas. Breve historia de la investigación en España. Marco legislativo. Los planes nacionales de investigación. La investigación en salud. Organismos públicos de investigación. El Instituto de Salud Carlos III y la promoción de la investigación en el entorno sanitario. La carrera de investigación en España

10. Sociología de la ciencia. Sociología interna y externa de la ciencia. Relación entre inversión y producción científica y tecnológica. Distribución geográfica de la producción científica. La ciencia española en el contexto internacional. Desviaciones de la ética científica

de las Profesiones Sanitarias [4] y 33/2011 General de Salud Pública [5] auspician el fomento de la investigación en el sistema sanitario y ponen toda la estructura asistencial al servicio de la investigación. Por otra parte, existe una estructura de dinamización de la investigación en salud, el Instituto de Salud Carlos III, mediante diferentes iniciativas formativas y de promoción de la investigación como, por ejemplo, los institutos de investigación sanitaria o biosanitaria [6].

Para alcanzar este nivel competencial, diferentes autores se han hecho eco, a lo largo de los últimos años, de la necesidad de que los médicos incorporen competencias de investigación a su currículo formativo y profesional, lo que debería incluir desde la investigación fundamental o básica hasta la investigación clínica, la investigación en servicios de salud o la investigación epidemiológica [7-12]. Así mismo, las guías de la Asociación para la Educación Médica en Europa (AMEE) estiman que un buen médico necesita competencias específicas y habilidades de investigación para convertirse en un médico práctico competente y un investigador en ciencias básicas y clínicas [13].

Sin embargo, no es menos cierto que el número de médicos que realizan investigación científica no crece al ritmo deseado, cuando no disminuye. Este problema no es únicamente español ni reciente. En 1979, James Wyngaarden, director de los Institutos Nacionales de Salud de Estados Unidos, ya advirtió de que el investigador clínico es una especie en peligro de extinción. A pesar de ello y de algunas medidas, la disminución del número de médicos que siguen una carrera científica ha continuado. De ahí que diversas universidades hayan implementado programas para incentivar la dedicación de los médicos a la investigación [14] emulando algunas experiencias ya consolidadas en Estados Unidos. La Universidad de Yale fue la primera que introdujo la realización de una tesis de investigación de pregrado en 1839 [15] y todos los estudiantes de esta universidad deben seguir programas tutorados de investigación en ciencias básicas, investigación clínica, ciencias sociales o investigación traslacional. Las universidades de Stanford y Duke $[16,17]$ mantienen programas de promoción de la investigación durante los estudios de grado desde 1959 y un número apreciable ha ido incorporando programas de intensificación en cuya oferta se incluyen actividades de investigación $[15,18,19]$.

Entre las estrategias seguidas por diferentes centros de educación superior se incluyen la intercalación de materias de ciencias en los estudios de medicina, de cursos monográficos orientados a la investigación, de seminarios y otras actividades. Estas iniciativas están vigentes en universidades de Estados Unidos [17,20,21], Latinoamérica [22-24], Europa [25-29], países árabes [30,31] y Canadá [32].

En la Universidad de Oviedo, aprovechando la adaptación de los títulos al Espacio Europeo de Educación Superior, hemos agrupado algunas competencias generales y específicas de la Orden ECI 332/ 2008 [33] a las de documentación, concepto de profesión, salud, enfermedad, enfermo y sistemas sanitarios, en una asignatura en la que los estudiantes reciben una aproximación a la investigación mediante contenidos teóricos, diseño experimental e historia de los descubrimientos científicos. Si bien la experiencia se ha realizado desde el inicio del Grado en Medicina en la Universidad de Oviedo 
(curso 2010-2011), en este artículo aportamos las actividades realizadas por los estudiantes en el curso 2017-2018 y su opinión sobre ellas.

\section{Sujetos y métodos}

\section{Organización de la asignatura}

La actividad se ha realizado con estudiantes de primer curso mediante la asignatura obligatoria 'Introducción a la medicina, documentación y método científico' del Grado en Medicina de la Universidad de Oviedo. Esta asignatura es de seis créditos ECTS, con 60 horas presenciales (58 de actividades y dos de examen escrito) y 90 horas no presenciales. Las horas presenciales se reparten en 22 clases expositivas, 10 de prácticas $(5 \times 2 \mathrm{~h}), 20$ de seminarios $(10$ $\times 2 \mathrm{~h}$ ), seis horas de tutoría grupal y dos de evaluación. Doce horas de clase y cuatro sesiones de seminarios (6 horas y 45 minutos) se dedican a actividades relacionadas con la investigación, incluyendo la presentación por el profesor de los objetivos y elaboración de los seminarios.

\section{Grupos de alumnos participantes}

Para las clases teóricas, los estudiantes (150-155 por curso) se distribuyen en dos grupos de unos 75 estudiantes. Para la realización de los seminarios se distribuyen en grupos de, aproximadamente, 40 alumnos. En cada grupo de seminario, los alumnos se distribuyeron en subgrupos de 4-5 alumnos, con un total de 32 subgrupos de trabajo.

\section{Organización de las actividades}

\section{Lecciones teóricas}

Las clases teóricas consisten en la exposición por el profesor de una serie de aspectos de aproximación a la investigación articulados en relación a los temas que se recogen en la tabla I.

\section{Seminarios}

Las sesiones de seminarios se distribuyen de la forma siguiente: una sesión de 45 minutos para la presentación por el profesor del objetivo general del seminario, los objetivos particulares y la forma de trabajo para abordar la tarea a realizar. También realiza un comentario participativo sobre un descubrimiento científico con repercusiones médicas. A partir de estos comentarios, los estudiantes de cada grupo disponen de dos semanas para realizar el ejercicio que deben presentar en la siguiente se- sión. Las siguientes dos sesiones (también de dos horas cada una) se destinan a trabajo de aula, dirigidos por el profesor, sobre comentario crítico de artículos de investigación, con especial énfasis en el diseño, y a la presentación del análisis (siguiendo las directrices sobre las que se ha trabajado en la sesión previa) de los trabajos asignados por el profesor a cada grupo. De esta última actividad, cada grupo de estudiantes debe redactar un texto sobre su trabajo siguiendo un formato estructurado (Tabla II). Este documento se incorpora al portafolio del campus virtual para computarlo dentro de la calificación global otorgada a este apartado, siendo su contribución neta de 0,75 puntos sobre 2,50.

\section{Desarrollo de los seminarios}

\section{Primera sesión. Presentación y objetivos}

En el primer seminario, el profesor presenta el objetivo general de la actividad, que consiste en identificar los aspectos relevantes del proceso de descubrimientos científicos relacionados con la enfermedad y la medicina mediante el análisis de casos que pueden considerarse ejemplares. En esta presentación, el profesor resalta los aspectos más relevantes que conducen a un descubrimiento científico y que servirán de guía para la tarea que los estudiantes deben realizar en el siguiente seminario. Las teorías clásicas establecen que los tres pilares de la investigación científica, en prácticamente todas las ramas del conocimiento, son tres: la realización de una observación que plantea una pregunta de investigación; la formulación de una hipótesis tentativa de resolución de la cuestión planteada mediante la propuesta de una asociación de variables en un sentido determinado, de tal manera que la alteración de una permita observar las modificaciones de las otras; y la realización de experimentos significativos que confirmen o rechacen la hipótesis, incorporando el nuevo conocimiento al marco teórico propio de las teorías concretas de cada rama de conocimiento $[34,35]$.

Teniendo en cuenta la complejidad que han ido adquiriendo los procesos de investigación en los últimos decenios, hemos preferido utilizar contextos más amplios en los que tengan cabida algunos de los aspectos que actualmente se consideran relevantes. Así, como se indica en el desarrollo del programa teórico recogido en la tabla I, el contexto de los descubrimientos incluye la observación y la hipótesis; el de verificación, todos los aspectos de diseño metodológico, y el de divulgación, la comunicación de los resultados tanto a la comunidad científica como a la ciudadanía. 
Tabla II. Esquema del desarrollo de los seminarios.

\begin{tabular}{|c|c|c|c|}
\hline \multirow{2}{*}{$\begin{array}{l}\text { Presentación } \\
\text { Primera sesión (tiempo: } 45 \text { min) }\end{array}$} & \multirow{2}{*}{$\begin{array}{l}\text { Descubrimientos científicos } \\
\text { Segunda sesión (tiempo: } 2 \text { h) }\end{array}$} & \multicolumn{2}{|c|}{ Diseño experimental } \\
\hline & & Tercera sesión (tiempo: 2 h) & Cuarta sesión (tiempo: 2 h) \\
\hline $\begin{array}{l}\text { Presentación por el profesor de } \\
\text { la orientación del seminario, de los } \\
\text { objetivos y de la forma de trabajo }\end{array}$ & $\begin{array}{l}\text { Todos los grupos presentan la tarea } \\
\text { realizada siguiendo el esquema } \\
\text { propuesto por el profesor }\end{array}$ & $\begin{array}{l}\text { Presentación por el profesor de } \\
\text { la orientación del seminario, de los } \\
\text { objetivos y de la forma de trabajo }\end{array}$ & $\begin{array}{l}\text { textos breves siguiendo el esquema } \\
\text { utilizado en la sesión anterior } \\
\text { Dos estudiantes presentan la tarea de }\end{array}$ \\
\hline $\begin{array}{l}\text { Realización, mediante un ejemplo, } \\
\text { del análisis de un descubrimiento } \\
\text { siguiendo el esquema que deben } \\
\text { desarrollar los estudiantes }\end{array}$ & $\begin{array}{l}\text { Cada grupo presenta la historia } \\
\text { de un descubrimiento científico } \\
\text { durante } 20-25 \text { minutos } \\
\text { Discusión o puntualizaciones }\end{array}$ & $\begin{array}{l}\text { Realización de ejemplos de transformación } \\
\text { de una observación en un problema de } \\
\text { investigación y de su concreción en los } \\
\text { apartados de un artículo científico }\end{array}$ & $\begin{array}{l}\text { análisis realizada con los dos artículos } \\
\text { científicos asignados por el profesor } \\
\text { Discusión o puntualizaciones } \\
\text { por estudiantes y profesor }\end{array}$ \\
\hline $\begin{array}{l}\text { El material analizado está disponible } \\
\text { en el campus virtual de la universidad }\end{array}$ & $\begin{array}{l}\text { por estudiantes y profesor } \\
\text { El ponente incorpora la } \\
\text { presentación al campus virtual }\end{array}$ & $\begin{array}{l}\text { Breve discusión en grupo, } \\
\text { presentación de observaciones } \\
\text { y discusión general en el seminario }{ }^{b}\end{array}$ & $\begin{array}{l}\text { Los ponentes de las últimas presentaciones } \\
\text { incorporan las tareas al campus virtual }{ }^{c}\end{array}$ \\
\hline
\end{tabular}

a Una vez concluida la sesión, los estudiantes pueden acceder a las tareas encomendadas a cada grupo que están disponibles en el campus virtual. b Una vez concluida la sesión, el profesor asigna dos artículos científicos publicados en revistas de investigación médica internacional; la mitad de los alumnos de cada sesión tienen asignado uno, y la otra mitad, el otro; también reciben textos breves en base a los cuales deben formular hipótesis, objetivos y metodología. ' Una vez concluida la sesión, los estudiantes deben incorporar un texto resumen de su actividad al campus virtual.

Algunos de los ejemplos analizados en cursos anteriores, en esta primera sesión, adaptados en la medida de lo posible al esquema anterior, han sido la asociación causal entre la 'materia pútrida' y la fiebre puerperal, el modelo observacional seguido por John Snow en la epidemia de cólera de 1848 en Londres, el ensayo clínico de James Lind en Salisbury en enfermos de escorbuto, el denominado 'experimento Karachi' sobre el mecanismo de contagio de la peste bubónica, o el ensayo de Gaspar Casal en el tratamiento de las 'fiebres mortíferas y la perineumonía' [36-40]. En el curso al que se refiere este trabajo, el ejemplo utilizado ha sido la asociación entre Helicobacter pylori y la úlcera gastroduodenal $[41,42]$ (Tabla III).

En 1979, Robin Warren, patólogo del hospital australiano de Perth, a la vista de unas bacterias que se suponían saprófitas y que colonizaban los nichos ulcerosos, se preguntó si, dada la frecuencia con que se observaba esta asociación, no tendrían algún papel en la fisiopatología de la úlcera, y en colaboración con el internista Barry J. Marshall y diversos servicios hospitalarios inició una serie de estudios encaminados a tal fin: caracterización del germen, ajuste a los postulados de Koch, desarrollo de un test de detección, validación clínica y respuesta a tratamiento a corto y largo plazo. La conclusión es que si bien puede haber otros factores que participen en la aparición de la úlcera duodenal, la infección por H. pylori parece ser el más notable de ellos. En
1994 fue aceptada la teoría infecciosa de la úlcera por los Institutos de Salud de Estados Unidos y en 2005 se concedió el Premio Nobel de Medicina a ambos científicos. Los aspectos más relevantes de la investigación se muestran en la tabla II.

En el campus virtual de la universidad está disponible, para consulta por el estudiante, la presentación y el ejercicio realizado por el profesor y una serie de orientaciones y recomendaciones para la elaboración de la tarea que los estudiantes deben presentar en el siguiente seminario.

\section{Segunda sesión. Presentaciones por los estudiantes} Para la realización del segundo seminario, cada grupo de alumnos tiene asignada la historia de un descubrimiento científico (Tabla III) con documentación relacionada para elaborar y presentar oralmente el trabajo siguiendo los apartados establecidos en la primera sesión.

El objetivo es identificar los aspectos relevantes del proceso de descubrimiento que han tenido lugar en descubrimientos científicos relacionados con la enfermedad y la medicina. En cuanto al procedimiento, cada subgrupo debe leer el documento asignado y extraer información relacionada con: época (fechas), ambiente científico de la época, protagonistas (origen, formación, características de la personalidad...), antecedentes del descubrimiento, identificación de una pregunta tentativa de investigación, proceso de análisis que siguió (tipo de razo- 
Tabla III. Aspectos relevantes de la metodología de investigación aplicados al descubrimiento de la asociación causal entre Helicobacter pylori y úlcera gastroduodenal.

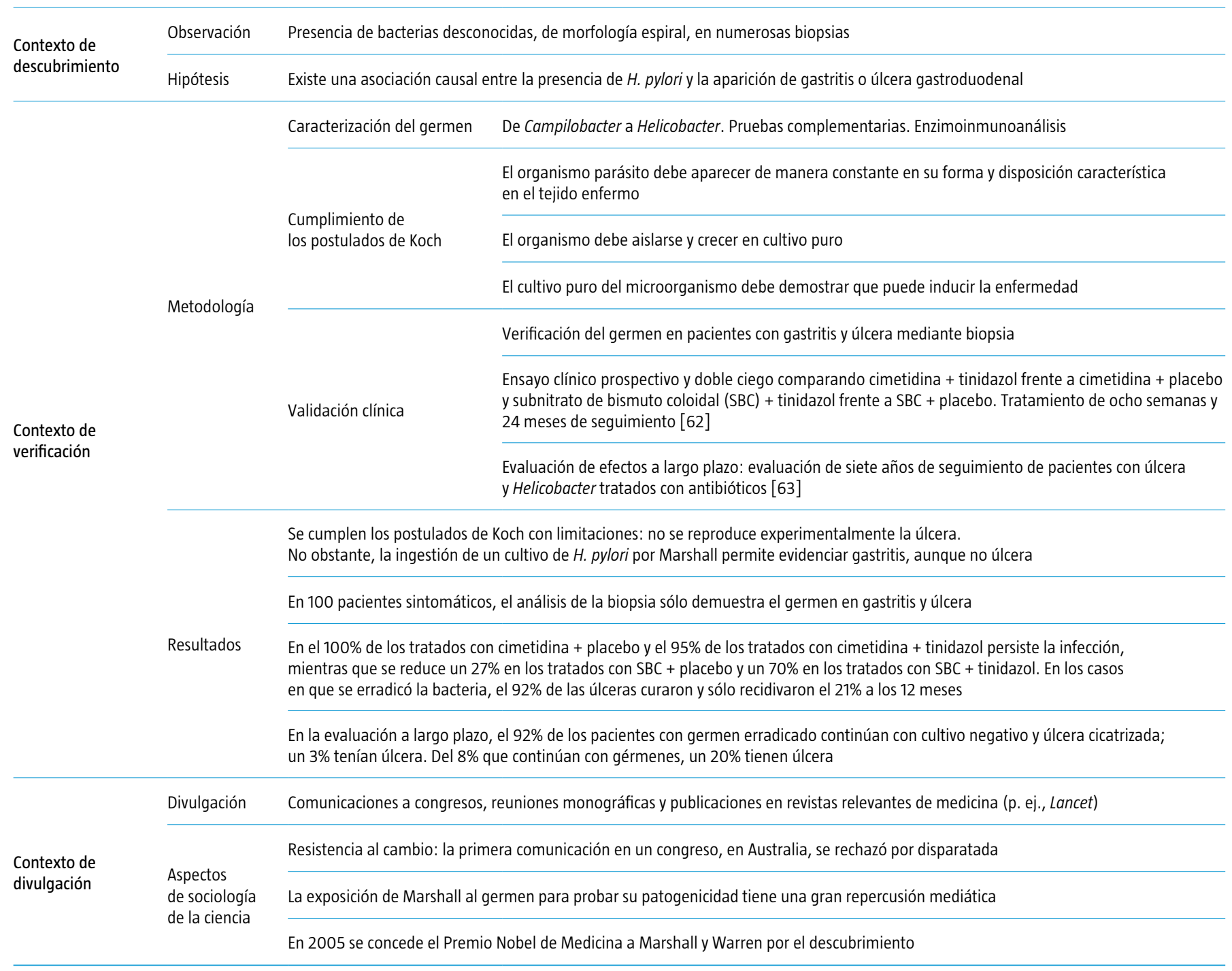

namiento y diseño de la demostración), cuál fue el proceso de divulgación y su repercusión, y vigencia actual del descubrimiento.

Con los datos extraídos, los estudiantes deben componer una breve historia en la que se relaten los aspectos científicos y, en la medida de lo posible, aspectos sociales que han estado implicados en el descubrimiento asignado. También deben preparar una presentación para exponer ante sus compañeros.

Entre el primer y este segundo seminario, los estudiantes dispusieron de dos semanas para la preparación de la tarea. Las exposiciones se realizan en una sesión de dos horas de duración en las que cada subgrupo dispone de 20-25 minutos y se reservan 5-10 minutos para comentarios por los estudiantes o precisiones del profesorado responsable del seminario. En cada sesión participan ocho subgrupos, de los que cuatro realizan la presentación de su trabajo.

Una vez finalizado el seminario, el alumno que ha realizado la exposición debe incorporar el documento expuesto al campus virtual. Este documento se ha utilizado para la evaluación cuantitativa de los diferentes apartados observados, así como las fuentes bibliográficas empleadas en la documentación.

\section{Tercera sesión}

En esta sesión se introduce a los alumnos en la formulación de preguntas de investigación o hipótesis 
de trabajo a partir de observaciones, para orientarlos sobre la forma de abordar las tareas de la cuarta sesión. Con este motivo, y dado que se trata de alumnos recién ingresados en el Grado en Medicina, se presenta un problema que no requiera conocimientos médicos previos, con el concurso de proyecciones de video. En este curso se ha utilizado un video [43] en el que un conocido actor cómico escenifica las posibles reacciones, por parte de un observador, al hallarse ante un paciente con parada cardiorrespiratoria, que informalmente introduce diferentes aspectos que pueden ser objeto de estudio. Posteriormente, otros dos videos muestran la reanimación cardiopulmonar 'clásica' y la llevada a cabo con compresión torácica sola, presentada por cardiólogos profesionales.

Tras la información expuesta, cada grupo de alumnos elabora una propuesta de identificación de los aspectos científicos involucrados en las escenas mostradas en los videos y los articulan como propuesta de investigación, formulando hipótesis, objetivos y metodología pertinente. Terminado este ejercicio grupal, un estudiante de cada grupo expone la tarea realizada, el profesor anota en la pizarra los elementos más relevantes de ella y plantea una discusión estructurada para resaltar los aciertos y las divergencias de apreciación entre los grupos.

En las siguientes sesiones, los estudiantes deberán aplicar el mismo desarrollo. El material de trabajo está disponible en el campus virtual de la universidad una semana antes de la sesión.

\section{Cuarta sesión}

En esta sesión se trata de desarrollar la competencia de los alumnos en la formulación de preguntas de investigación, hipótesis, objetivos de investigación y metodología a partir de textos sugerentes de problema sanitarios, así como la capacidad de identificar estos apartados (además de los de resultados y discusión) en artículos originales de investigación.

Para ello se les asigna textos breves (diferentes para cada subgrupo), a partir de los cuales han de formular una pregunta de investigación y establecer hipótesis, objetivos y metodología para desarrollarla, y dos artículos científicos originales sencillos (la mitad de los subgrupos recibe un artículo diferente del de la otra mitad), para que extraigan información concisa de cada uno de los apartados del mismo.

De ambas partes de esta tarea, los alumnos elaboran un documento escrito, que incorporan al campus virtual para su evaluación, y una presentación de PowerPoint, que comentan en el transcurso del seminario. La tarea sobre los textos breves es presentada por cada subgrupo durante cinco minutos y, posteriormente, es sometida a discusión por el resto de estudiantes y por el profesor. El análisis de las publicaciones es presentado por un único estudiante integrante de uno de los subgrupos que han trabajado en el artículo asignado y se sigue de unos minutos de comentarios. Ambas exposiciones son objeto de puntuación.

Terminada esta sesión, cada subgrupo debe incorporar al portafolio del campus virtual de la asignatura un texto que recoja sus actividades en los apartados de la cuarta sesión para su calificación. Un esquema del procedimiento se muestra en la tabla II.

\section{Instrumentos de evaluación}

Se han utilizado distintos instrumentos para evaluar los aspectos relevantes de la actividad de los estudiantes, y la satisfacción de éstos.

\section{Calificación de la tarea presentada en los seminarios}

Se realiza siguiendo un cuestionario estructurado que incorpora los siguientes epígrafes: contenido del trabajo, organización de la presentación, claridad de la presentación, ritmo y duración de la presentación, contacto visual y lenguaje no verbal, medios audiovisuales utilizados, y defensa y argumentación en el coloquio. A cada uno de estos epígrafes se le asigna una escala de 1 a 4 y se realiza la media de la calificación obtenida en todos los apartados. Esta calificación es la que recibe cada uno de los alumnos del subgrupo en ese seminario. En el acto de presentación, el profesor toma las notas pertinentes para establecer esta calificación. Si fuera necesaria alguna precisión añadida, se consulta el texto incorporado al campus virtual. La contribución de esta actividad a la calificación final de los estudiantes es de 1 punto sobre 10 .

\section{Calificación del texto escrito consecutivo al análisis crítico de un artículo de investigación}

Se atiende a los siguientes aspectos: inclusión del título del artículo, identificación del subgrupo de seminario y miembros del mismo, contenido descriptivo, análisis crítico, conclusiones y referencias bibliográficas. La contribución de esta actividad a la calificación final de los estudiantes es de 0,75 puntos sobre 10.

\section{Cuantificación de la opinión de los alumnos sobre el valor formativo de la actividad}

Se diseña una encuesta de satisfacción en la que se pregunta por los tres aspectos que se abordan en el 
curso (lecciones teóricas, seminarios sobre descubrimientos científicos y sobre crítica de artículos de investigación, y diseño de investigación). Los estudiantes cumplimentaron la encuesta de forma voluntaria y anónima al final del curso. Se les pidió que calificaran (de 0 a 5 , teniendo en cuenta que 0 indica su total desacuerdo y 5 su total acuerdo con la opción) el interés que suscitaron en ellos los aspectos cuestionados en la misma.

\section{Estadística}

Los datos se presentan como valores de la media \pm error estándar de la media. Se ha realizado el análisis de la varianza (ANOVA) de un factor para evaluar si existían o no diferencias entre las calificaciones asignadas a los ítems en las distintas actividades, aplicando el test de Bonferroni para identificar entre cuáles la diferencia era significativa $(p<0,05)$.

\section{Resultados}

En la experiencia han participado los estudiantes del curso académico 2017-2018 (unos 150). Las clases teóricas han tenido una asistencia descendente a medida que se avanzaba en el curso. En el tiempo previsto, se han impartido todos los temas programados sin incidencias notables. Los temas de iniciación abordados, como se muestra en la tabla I, se relacionan con el proceso de documentación y con diferentes aspectos de la metodología de la investigación científica en general y de la investigación en medicina en particular.

Respecto a los seminarios, la asistencia en ambos cursos ha sido elevada dada su naturaleza obligatoria; se presentaron todas las tareas asignadas y se incorporaron al campus virtual tanto las presentaciones en PowerPoint como la narración del último seminario destinado al diseño experimental.

Las narraciones relacionadas con descubrimientos científicos se muestran en la tabla IV y aluden a descubrimientos relevantes en fisiología (p. ej., descubrimiento de hormonas y vitaminas), patología (p. ej., identificación de agentes infecciosos y de mecanismos de transmisión de enfermedades) o terapéutica (p. ej., desarrollo de analgésicos y antibióticos), en los que se pueden poner de manifiesto, en mayor o menor medida, los aspectos metodológicos y sociológicos de la investigación científica. También hay algunos ejemplos del desarrollo de tecnologías aplicadas a la investigación o a la terapéutica, como los cultivos celulares, las células madre, la clonación o los trasplantes. En la descripción de sus
Tabla IV. Relación de descubrimientos científicos que han sido objeto de análisis por los estudiantes (los textos ofrecidos a los estudiantes han sido tomados de las referencias [47-56]).

\begin{tabular}{lll}
\hline Antibióticos & Digital & Quinina \\
Antipiréticos & Efecto anestésico de la cocaína & Psicoanálisis \\
Barbitúricos & El colesterol & Reflejos condicionados \\
Circulación sanguínea & Gripe: agente y contagio & Sida \\
Células madre & Hormonas & Sulfamidas \\
Clonación & La lepra & Transmisión del cólera \\
Código genético & La peste & Trasplantes \\
Control del dolor & La radiología & Tuberculosis \\
Control de la senescencia & La radioterapia & Vacunación \\
Control de la sífilis & Malaria (enfermedad y transmisión) & Vitaminas \\
Cultivos celulares & Purificación de la morfina &
\end{tabular}

tareas, los estudiantes se han ajustado mayoritariamente al guion de análisis propuesto en las instrucciones. Las calificaciones medias obtenidas por los estudiantes de los 32 subgrupos, teniendo en cuenta los diferentes aspectos analizados en la presentación, fue de 3,7 $\pm 0,07$ puntos sobre 4 .

El ejemplo utilizado para el ejercicio general de análisis de una publicación se ha relacionado con la resucitación cardiopulmonar [43]. Los artículos utilizados para presentación por los estudiantes y discusión en cada sesión de seminarios han sido dos: uno, relacionado con la ingesta de alcohol y la aparición de ictus [44], y otro, sobre la relación entre la disminución de peso y el control de la diabetes y la mortalidad cardiovascular [45]. Los diseños de investigación elaborados por los estudiantes a partir de pequeños relatos han sido los ocho siguientes: flúor y alteraciones cognitivas, marcadores genéticos de longevidad, ingesta de alcohol y enfermedad mental, calidad del aire y patología cardiovascular aguda, curcumina y trastornos psiquiátricos, ayuno y longevidad, consumo de café y salud, y frutos secos y riesgo cardiovascular.

Las calificaciones obtenidas por los estudiantes en las presentaciones de sus comentarios sobre los trabajos asignados fueron de 3,9 $\pm 0,3$ puntos, y la obtenida en la descripción del diseño experimental, de 3,6 $\pm 0,2$ puntos sobre 4 .

\section{Resultados de la encuesta}

Hemos recogido la opinión de los estudiantes sobre la contribución formativa de las actividades, su satisfacción y la idoneidad de las mismas para poner de manifiesto algunos aspectos relacionados con la investigación en general y la aplicada a la medicina. La encuesta se ha cumplimentado a final de curso. El número de encuestas válidas ha sido de 128 (84,21\% de los matriculados). 
Tabla V. Resultados de la encuesta del curso 2017-2018. Los estudiantes deben cuantificar (de 0 a 5) la utilidad otorgada a las tres actividades para valorar su contribución a los aspectos incorporados al cuestionario.

\begin{tabular}{|c|c|c|c|c|}
\hline & Teoría & Descubrimiento & Diseño experimental & $p<0,05$ \\
\hline Aspectos relevantes de los conceptos de ciencia e investigación & $3,74 \pm 0,11$ & $3,96 \pm 0,08^{a}$ & $3,63 \pm 0,10$ & 2 frente a 3 \\
\hline Diferencias entre ciencias y pseudociencias & $3,94 \pm 0,10^{a}$ & $3,04 \pm 0,12$ & $2,96 \pm 0,14$ & $\begin{array}{l}1 \text { frente a } 3, \\
1 \text { frente a } 2\end{array}$ \\
\hline Diferentes apartados del proceso de investigación & $4,29 \pm 0,09^{a}$ & $3,84 \pm 0,10$ & $3,98 \pm 0,10$ & 1 frente a 2 \\
\hline Medicina como una ciencia con entidad propia & $4,01 \pm 0,11^{\mathrm{a}}$ & $3,79 \pm 0,11$ & $3,59 \pm 0,11$ & 1 frente a 3 \\
\hline $\begin{array}{l}\text { Importancia de cualidades actitudinales } \\
\text { (curiosidad, interés, perseverancia...) en la investigación }\end{array}$ & $4,14 \pm 0,09$ & $4,15 \pm 0,09$ & $3,94 \pm 0,10$ & NS \\
\hline Importancia del azar en los descubrimientos científicos & $3,76 \pm 0,10$ & $3,97 \pm 0,10$ & $3,45 \pm 0,11$ & 2 frente a 3 \\
\hline $\begin{array}{l}\text { El método científico puede aplicarse a la resolución } \\
\text { de problemas que afectan a los enfermos }\end{array}$ & $4,13 \pm 0,09$ & $4,08 \pm 0,09$ & $4,02 \pm 0,10$ & NS \\
\hline Las respuestas obtenidas en la investigación clínica son probabilísticas & $4,07 \pm 0,10^{a}$ & $3,76 \pm 0,11$ & $3,66 \pm 0,12$ & 1 frente a 3 \\
\hline $\begin{array}{l}\text { La discusión en grupo aporta una mejor comprensión } \\
\text { de los problemas biomédicos y su abordaje científico }\end{array}$ & $3,75 \pm 0,11^{\mathrm{a}}$ & $4,21 \pm 0,09$ & $4,28 \pm 0,08$ & $\begin{array}{l}1 \text { frente a } 2, \\
1 \text { frente a } 3\end{array}$ \\
\hline Importancia formativa de redactar un breve texto científico & $3,40 \pm 0,12$ & $3,68 \pm 0,11$ & $3,87 \pm 0,11^{\mathrm{a}}$ & 3 frente a 1 \\
\hline La ciencia es una tarea colectiva & $4,09 \pm 0,10$ & $4,20 \pm 0,10$ & $4,17 \pm 0,10$ & NS \\
\hline La comunicación de los resultados contribuye al progreso de la ciencia & $4,31 \pm 0,08$ & $4,33 \pm 0,08$ & $4,22 \pm 0,09$ & NS \\
\hline Necesidad de que los médicos realicen investigación científica & $4,15 \pm 0,10$ & $4,16 \pm 0,09$ & $4,14 \pm 0,09$ & NS \\
\hline La investigación científica soluciona problemas de salud & $4,36 \pm 0,08$ & $4,41 \pm 0,07$ & $4,27 \pm 0,08$ & NS \\
\hline
\end{tabular}

NS: no diferencias en ANOVA. a $p<0,05$; test de Bonferroni.

En todos los aspectos cuestionados, las puntuaciones otorgadas a las tres actividades están por encima de los 3 puntos sobre 5 (Tabla V), siendo la puntuación global media de 4,01 $\pm 0,07$ para las lecciones teóricas, de 3,97 $\pm 0,09$ para el seminario sobre los descubrimientos científicos y de 3,87 $\pm 0,1$ puntos para las tareas de diseño experimental. El análisis de la varianza indica que, en opinión de los estudiantes, hay una contribución diferenciada de las tres estrategias docentes. Un posterior análisis mediante el test de Bonferroni indica que estas diferencias pueden identificarse en ocho de las 14 cuestiones planteadas (Tabla V).

Por último, se formularon dos cuestiones para las tres actividades encaminadas a conocer la satisfacción general de los estudiantes y el valor que le conceden en la formación del médico. En el primer caso, las calificaciones fueron de 3,46, 3,92 y 3,76 puntos, mientras que en el segundo los estudiantes otorgaron 3,39, 3,65 y 3,44 puntos, respectivamente, para las lecciones teóricas y los seminarios sobre descubrimientos científicos y diseño experimental. En ambos casos, los seminarios sobre descubrimientos científicos aportan la mayor satisfacción y son los que, tentativamente, tendrían una mayor contribución a la formación del médico.

\section{Discusión}

El objetivo de adquirir una sólida formación científica implica que el médico debe tomar contacto con las actividades de investigación de la forma más precoz posible, en la que la formación de grado puede ser el primer paso de iniciación a la investigación, como ocurre en otros países [17,20-32,46]. En buena parte de estas iniciativas, se constata que la dedicación a la investigación debe iniciarse de 
forma precoz y que generalmente es bien recibida. Entre las estrategias seguidas se incluyen, entre otras, cursos monográficos sobre investigación, seminarios y otras actividades a las que podemos considerar que, en líneas generales, se ajusta nuestra experiencia.

Al tratarse de una asignatura obligatoria, todos los estudiantes han participado en las actividades. Como es frecuente, la asistencia a las clases teóricas decrece a lo largo del curso; en cambio, la presencia en los seminarios es completa porque se consideran obligatorios. Dado que se trata de un curso de introducción, los contenidos teóricos son presentados a un nivel de inicio con la finalidad de que resulten más comprensibles las presentaciones que reciban en asignaturas posteriores. Así, por ejemplo, los estudiantes no se exponen a una descripción pormenorizada de la estadística (reciben sus conocimientos en una asignatura que se imparte de forma paralela), sino que se limita a la enunciación de su papel en el diseño de una investigación y del significado real de sus aportaciones a la explicación que los experimentos aportan a la resolución del problema incluido en una hipótesis: el resultado como hecho científico, el valor de la significación (valor de $p$ ) o la diferencia entre la significación estadística y la significación clínica.

Para la propuesta de seminarios sobre descubrimientos científicos hemos utilizado textos actuales y otros escritos hace muchos años [47-56] con la idea de que los estudiantes sean conscientes de que 'el mundo no comienza con cada generación, es un fluir continuo y si no advertimos que provenimos de un pasado, difícilmente podemos crear un futuro significativo' [57]. Naturalmente que los temas propuestos pueden ser objetados y considerados adecuados o no, pero tienen la ventaja de exponer a los estudiantes (en muchos casos por primera vez) a lecturas sobre aspectos de la generación de conocimiento aplicable en su futura profesión. El hecho de que los estudiantes trabajen con un esquema de actuación sobre los aspectos potencialmente implicados en los descubrimientos científicos (Tabla III) facilita la realización de la tarea. No obstante, los profesores que decidan secundar esta experiencia pueden sustituirlos por aquellos que consideren más adecuados o próximos a su quehacer o a su idea educativa, como hemos hecho nosotros.

Las tareas abordadas en el seminario sobre lectura crítica de literatura científica y diseño experimental ponen en contexto práctico, por un lado, algunos de los aspectos de la documentación científica (que también forma parte de la asignatura en la que se inscriben las actividades) y, por otro, ofre- cen además la oportunidad de que, a partir de un texto breve, los estudiantes identifiquen un problema de investigación, formulen una hipótesis de resolución y elaboren un esquema metodológico para verificarla. De esta forma, los estudiantes son expuestos, a nivel de iniciación, a toda una serie de aspectos de teoría y diseño de investigaciones que pueden llevar a cabo en cursos sucesivos mediante la oferta de asignaturas optativas relacionadas con investigación que ofrece el grado [58]. Además, al tratarse de tareas de grupo que se exponen en sesiones con estudiantes, se favorece el desarrollo de competencias transversales de comunicación y discusión estructurada en la que el tutor actúa como moderador.

Al finalizar el último seminario, volvemos a recordar que todo el proceso comienza con una observación creativa y, para reforzar esta apreciación, les hacemos partícipes del pensamiento de Barry Marshall respecto al descubrimiento del papel de H. pylori en la patogenia de la úlcera: 'sólo tuvimos que convencer a todos los demás científicos del mundo de lo que veíamos [...], no podían negarme que la bacteria estaba porque yo la veía todos los días en mi laboratorio [...]. Yo, simplemente, me di cuenta de lo que pasaba, porque seguro que no fui el primero en ver tejidos de estómago con la bacteria. Simplemente, me di cuenta de que ahí pasaba algo [...]. El mérito estuvo en ir más allá y darse cuenta de las implicaciones que eso tenía' [59].

Las calificaciones obtenidas por los estudiantes son elevadas $(3,71 \pm 0,07,3,9 \pm 0,3$ y 3,6 $\pm 0,2$ sobre 4$)$, como corresponde al nivel de corte de acceso al grado, y son similares a las obtenidas por estudiantes de esta misma asignatura en otras actividades $[60,61]$.

Los estudiantes consideran que las actividades realizadas son útiles para entender el valor del método científico en la elaboración de la ciencia, que puede aplicarse a la resolución de problemas que afectan a los pacientes y soluciona problemas de salud, y que la medicina tiene entidad propia dentro de la investigación científica. También consideran que la comunicación científica es importante en ciencia. En todo caso, aunque las valoraciones son muy próximas, un análisis pormenorizado sugiere que algunas actividades ofrecen ventaja respecto a otras en la percepción de los estudiantes. Globalmente, los estudiantes se consideran satisfechos con las actividades realizadas y piensan que pueden contribuir a la formación del médico.

En consecuencia, consideramos que la organización de este tipo de acciones puede iniciar a los estudiantes de medicina en las tareas de investigación y contribuir no sólo a la formación de médicos cien- 
tíficos, sino también al fomento de la vocación de médicos investigadores.

\section{Bibliografía}

1. Rodríguez-Sendín JJ. Definición de 'profesión médica', 'profesional médico/a' y 'profesionalismo médico'. Educ Med 2010; 13: 63-6.

2. Ley $14 / 1986$, de 25 de abril, General de Sanidad. Boletín Oficial del Estado 1986; 102: 15207-24.

3. Ley $16 / 2003$, de 28 de mayo, de Cohesión y Calidad del Sistema Nacional de Salud. Boletín Oficial del Estado 2003; 128: 20567-88.

4. Ley 44/2003, de 21 de noviembre, de Ordenación de las Profesiones Sanitarias. Boletín Oficial del Estado 2003; 280: 41442-58

5. Ley 33/2011, de 4 de octubre, General de Salud Pública. Boletín Oficial del Estado 2011; 240: 104593-626.

6. Real Decreto $279 / 2016$, de 24 de junio, sobre acreditación de institutos de investigación biomédica o sanitaria. Boletín Oficial del Estado 2016; 161: 47272-83.

7. Rodés J. La experiencia del Hospital Clínico de Barcelona: integración de la Facultad de Medicina -IDIBAPS - hospital universitario. Educ Med 2007; 10: 202-6.

8. Cardellach F, Vilardell M. Hacia el perfil de médico que necesita la comunidad. Med Clin (Barc) 2006; 127: 136-8.

9. Millán J. El conocimiento: sustrato sine qua non del médico en el sistema sanitario. Educ Med 2007: 10 (Supl 2): 20-2.

10. Torres A. El médico como investigador. Educ Med 2007; 10 (Supl 2): 27-30.

11. Giménez-Amaya JM. Necesidad de médicos dedicados a la docencia universitaria y a la investigación biomédica básica. Educ Med 1999; 2: 192-3.

12. Giménez-Gómez N, Pedrazas-López D, Medina-Rondón E, Dalmau-Juanola D; Grupo de Estudio sobre la Formación en Investigación. Formación en investigación: autopercepción de los profesionales sobre sus necesidades. Med Clin (Barc) 2009; 132: 112-7.

13. Laidlaw A, Aiton J, Structhers J, Guild S. Developing research skills in medical students: AMEE Guide No. 69. Med Teach 2012; 34: 754-71.

14. Fishleder AJ, Henson LC, Hull AL. Cleveland Clinic Lerner College of Medicine: an innovative approach to medical education and the training of physician investigators. Acad Med 2007; 82: 390-6.

15. Green EP, Borkan JM, Pross SH, Adler SR, Nothnagle M, Parsonnet J, et al. Encouraging scholarship: medical school programs to promote student inquiry beyond the traditional medical curriculum. Acad Med 2010; 85: 409-18.

16. Grochowski CO, Halperin EC, Buckley E. A curricular model for the training of physician scientists: the evolution of the Duke University School for medicine curriculum. Acad Med 2007; 82: 375-82.

17. Laskowittz DT, Drucker RP, Parsonnet J, Cross PC, Gesundheit N. Engaging students in dedicated research and scholarship during medical school: the long-term experiences at Duke and Stanford. Acad Med 2010; 85: 419-28.

18. Parsonnet J, Gruppuso PA, Kanter SL, Boninger M. Required vs elective research and in-depth scholarship programs in the medical student curriculum. Acad Med 2010; 85: 405-8.

19. Rosemblatt RA, Desnick L, Corrigan C, Keerbs A. The evolution of a required research program for medical students at the University of Washington School of medicine. Acad Med 2006; 81: 877-81.

20. Solomon SS, Tom SC, Pichet J, Wasserman D, Powers AC. Impact of medical student research in the development of physician-scientists. J Invest Med 2003; 51: 149-56.

21. George P, Green EM, Park YS, Gruppuso PA. A 5-year experience with an elective scholarly concentrations program. Med Educ Online 2015; 20: 29278.

22. Molina-Ordóñez J, Huamaní C, Mayta-Tristán P. Apreciación estudiantil sobre la capacitación universitaria en investigación: estudio preliminar. Rev Peru Med Exp Salud Pública 2008; 25 325-9.

23. Arellano-Sacramento C, Hermoza-Moquillaza RV, ElíasPodestá M, Ramírez-Julca M. Actitud hacia la investigación de estudiantes universitarios en Lima, Perú. FEM 2017; 20: 191-7.

24. Ríos-González CM. Capacitación en investigación científica en estudiantes de medicina. FEM 2017; 20: 313.

25. Hren D, Lukić IK, Marusić A, Vodopivec I, Vujaklija A, Hrabak M, et al. Teaching research methodology in medical schools: students' attitudes towards and knowledge about science. Med Educ 2004; 38: 81-6.

26. Stubbs TA, Lightman EG. Mathieson P. Is it intelligent to intercalate? A two centre cross-sectional study exploring the value of intercalated degrees, and the possible effects of the recent tuition fee rise in England. BMJ Open 2013; 3: e002193.

27. Mahesan N, Crichton S, Sewell H, Howell S. The effect of an intercalated BSc on subsequent academic performance. BMC Med Educ 2011; 11: 76.

28. Boyle SE, Cotton SC, Myint PK, Hold GL. The influence of early research experience in medical school on the decision to intercalate and future career in clinical academia: a questionnaire study. BMC Med Educ 2017; 17: 245.

29. Möller R, Shoshan M. Medical students' research productivity and career preferences; a 2-year prospective follow-up study. BMC Med Educ 2017; 15: 51.

30. Khan H, Khan S, Iqbal S. Knowledge, attitudes and practices around health research: the perspective of physicians-in-training in Pakistan. BMC Med Educ 2009; 9: 46.

31. Amgad M, Tsui MMK, Liptrott SJ, Shash E. Medical student research: an integrated mixed-methods systematic review and meta-analysis. PLoS One 2015; 10: e.0127470.

32. Chang Y, Ramnanan CJ. A review of literature on medical students and scholarly research: experiences, attitudes, an outcome. Acad Med 2015; 90: 1162-73.

33. Orden $E C I / 332 / 2008$, de 13 de febrero, por la que se establecen los requisitos para la verificación de los títulos universitarios oficiales que habiliten para el ejercicio de la profesión de médico. Boletín Oficial del Estado 2008; 40: 8351-5.

34. Bernard C. Introducción al estudio de la medicina experimental. Madrid: Crítica; 2005

35. Chalmers AF. ¿Qué es esa cosa llamada ciencia? Madrid: Siglo XXI; 2006.

36. López-Cerezo JA. El triunfo de la antisepsia. Un ensayo en filosofía naturalista de la ciencia. México DF: Fondo de Cultura Económica; 2008.

37. Hempel CG. Filosofía de la ciencia natural. Madrid: Alianza Editorial; 1999.

38. Casal G. Experimentos, tocantes al uso de los vegigatorios, en la generalísima epidemia de fiebres ardientes, y perineumonías, que infestaron este País el Otoño de el año de 1735 y parte del Invierno de 36. In: Historia natural y médica del Principado de Asturias. Oviedo: Consejería de Educación, Cultura y Deportes. Servicio de Publicaciones del Principado de Asturias; 1988. p. 242-53.

39. Deville P. Peste y cólera. Barcelona: Anagrama; 2014.

40. Brown SR. Escorbuto. Cómo un médico, un navegante y un caballero resolvieron el misterio de la peste de las naos. Barcelona: Juventud; 2005.

41. Thagard P. How scientists explain disease. Princeton: Princeton University Press; 1999.

42. Fresnedo RM, Hidalgo A. El cambio de paradigma en la concepción y etiopatogenia de la gastritis y la úlcera péptica tras el descubrimiento de Helicobacter pylori. Rev Esp Invest Quirúrgicas 2016; 19: 119-27.

43. Hüpfl M, Selig HF, Nagele P. Chest-compression-only versus standard cardiopulmonary resuscitation: a meta-analysis. Lancet 2010; 376: 1552-7.

44. Caicoya M, Rodríguez T, Corrales C, Cuello R, Lasheras C. Alcohol and stroke: a community case-control study in Asturias, Spain. J Clin Epidemiol 1999; 52: 677-84.

45. Franco M, Bilal U, Orduñez P, Benet M, Morejón A, Caballero B, et al. Population-wide weight loss and regain in relation to diabetes burden and cardiovascular mortality in Cuba 1980- 
2010: repeated cross sectional surveys and ecological comparison of secular trends. BMJ 2013; 346: f1515.

46. Ricoy JR, Carrasco M, Clavería LR. Educación médica e investigación. Med Clin (Barc) 1999; 112: 259-63.

47. Alinovi M. Historia de las epidemias. Madrid: Capital Intelectual; 2009.

48. Carranza FA. Héroes de la medicina. Buenos Aires: AUDEBA; 2008.

49. Friedman M, Friedman GM. Los diez mayores descubrimientos de la medicina. Barcelona: Paidós Ibérica; 1999.

50. Johnson G. Los diez experimentos más hermosos de la ciencia Barcelona: Ariel; 2008.

51. Macip S. Inmortales y perfectos. Barcelona: Destino; 2008.

52. Macip S. Las grandes plagas modernas. Barcelona: Destino; 2010.

53. Montagnier L. Las batallas de la vida. Madrid: Alianza Editorial; 2009.

54. Silverman M. Drogas mágicas. 3 ed. Buenos Aires: Editorial Sudamericana; 1944

55. Valpuesta JM. A la búsqueda del secreto de la vida. Una breve historia de la biología molecular. Madrid: Hélice/CSIC; 2008.

56. De Kruif P. Los cazadores de microbios. México DF: Porrúa; 2011.

57. Etcheverry GJ. El desafío de formarse como médico. In VV. AA.
¿Por qué ser médico hoy? Buenos Aires; Libros del Zorzal: 2009. p. 19-30.

58. Grado en Medicina por la Universidad de Oviedo. URL: http://www.uniovi.es/estudios/grados/-/asset_publisher/ X5CYKURHdF1e/content/grado-en-medicina-2014?redirec $\mathrm{t}=\% 2$ Festudios\%2Fgrados.

59. De Benito E. No podían negarme que la Helicobacter estaba La veía a diario. El País, 19 de diciembre de 2011. URL: https:// elpais.com/sociedad/2011/12/19/actualidad/1324299101_ 754723.html.

60. Hidalgo A, Bordallo J, Sánchez M, Cantabrana B. Protagonismo de los alumnos en el aprendizaje. Una experiencia en el primer curso de medicina. Educ Med 2012; 15: 213-9.

61. Cantabrana B, Hidalgo A. Una experiencia de aproximación a la clínica en el primer curso del Grado en Medicina en la Universidad de Oviedo. FEM 2018; 21: 31-9.

62. Marshall BJ, Goodwin CS, Warren JR, Murray R, Blincow ED, Blackbourn SJ, et al. Prospective double-blind trial of duodenal ulcer relapse after eradication of Campylobacter pylori. Lancet 1988; 2: 1437-42.

63. Forbes GM, Glaser ME, Cullen DJ, Warren JR, Christiansen KJ, Marshall BJ, et al. Duodenal ulcer treated with Helicobacter pylori eradication: seven-year follow up. Lancet 1994; 343: 258-60. 